\title{
1-D Feature Extraction Using a Dispersive Scattering Center Parametric Model
}

\author{
Dane F. Fuller, Andrew J. Terzuoli, Peter J. Collins, Robert Williams \\ Air Force Institute of Technology; Air Force Research Laboratory
}

\section{INTRODUCTION}

Automatic object recognition, the ability for a machine to classify an object it has detected, is an area of continual interest. The classification of objects requires extraction of discriminatory information, and this process is called feature extraction. The extractable features are inextricably tied to the type of sensor used, and this work addresses one-dimensional, High Resolution Radar (HRR).

Several approaches have been proposed for feature extraction in HRR. The most straightforward approach lies in using the magnitude of range bins in range profiles as features. This approach generally denotes template matching [1]; however, template matching is not an optimal approach because it includes many noisy range bins as features. A different approach is based on finding sinusoids in noise by a sum-of-damped exponentials (DE) model. These superresolution techniques include Prony [2-5], Matrix Pencil [6], and many others. The features of the DE-based scattering models, range and amplitude, have been used for feature extraction, but the DE model simplistically assumes that all scatterering centers on the object have the frequency response of an ideal point scatterer. One high potential improvement to the damped exponential model lies in estimating the frequency response of each scatterering center uniquely. This improvement is based on the Geometric Theory of Diffraction (GTD) [7] and the Uniform Theory of Diffraction (UTD) [8] and includes electromagnetic scattering theory as a basis for scattering center modeling. Research in adapting DE models to include GTD/UTD has been performed [ 9 , 10], but has had limited implementation in object recognition problems. This work provides analysis and a proof-of-concept for implementing the GTD/UTD-based dispersive scattering center (DSC) model to perform object recognition. The DSC model presented here is based directly upon Potter's work at The Ohio State University [10].

\section{THE DISPERSIVE SCATTERING CENTER MODEL}

GTD as developed by Keller [7] predicts that at high frequencies, the backscattered field is related to the incident field and appears to originate from a discrete set of independent scattering centers. The backscattered field is given by

$$
\overrightarrow{\mathbf{E}}^{s}(f, z) \approx \frac{\overrightarrow{\mathbf{E}}_{0}}{|z|} e^{j \frac{2 \pi f}{c} z} \sum_{m=1}^{M} \phi_{m, f} e^{-j \frac{4 \pi f}{c} \hat{z} \cdot \overline{\mathbf{r}}_{m}}
$$

U.S. Government Work Not Protected by U.S. Copyright 
where $|z|$ is the spreading factor, $\phi_{m}, f$ is the frequency dependent magnitude of the scatterer, and $\mathrm{rm}$ is the distance to the $\mathrm{m} / \mathrm{h}$ scattering center from the zero phase reference.

Assuming a given polarization and a normalized field, the GTD/UTD-based dispersive scattering center (DSC) model is given by [10]

$$
\mathrm{E}(f)=\sum_{m=1}^{M} A_{m}\left(j \frac{f}{f_{c}}\right)^{\alpha_{m}} e^{-j \frac{4 \pi}{c} r_{m}}
$$

Although one also estimates the complex magnitude, $A_{m}$, the parameters of interest for object recognition are the type parameter, $\alpha_{m}$ and the range parameter, $r_{m}$. The range parameter gives the location of the scatterer, and the type parameter describes the frequency response of the scatterer. The type parameter is an integer multiple of $1 / 2$ as given by GTD/UTD. A listing of type parameters and their associated scattering geometries is provided in Table 1.

Table 1. Type Parameters and Their Associated Scattering Geometries [10]

Value of $\alpha$
\begin{tabular}{|c|l|}
\hline 1 & Example Scattering Geometries \\
\hline $1 / 2$ & flat plate specular; dihedral \\
\hline 0 & $\begin{array}{l}\text { singularly curved surface } \\
\text { straight edge specular }\end{array}$ \\
\hline$-1 / 2$ & edge diffraction \\
\hline-1 & comer diffraction \\
\hline less than -1 & $\begin{array}{l}\text { multi-bounce; unresolved or } \\
\text { combined mechanisms }\end{array}$ \\
\hline
\end{tabular}

The advantage of the GTD/UTD-based DSC model is that certain types of scattering (i.e. dihedral, curved edge, straight edge, point scatterer, etc.) will display a continuous change in magnitude with change in frequency. Therefore, as was stated before, one can essentially model an object as a sum of independent scattering geometries. From Equation 2, the magnitude of scatterer $m_{0}$ is proportional to $\left(j f / f_{c}\right)^{\alpha}$ where $f_{c}$ is the center frequency of the "chirped" radar pulse. Therefore, the frequency response of each scatterer determines the scattering geometry.

\section{PROOF OF CONCEPT}

An example fifth-order scatterer estimation for an aircraft at 5 degrees azimuth and 0 degrees elevation is provided in Table 2 . For this feature extraction example, a fifth order DSC model has ten parameters of interest, a type and range parameter for each scattering center estimated. These ten parameters may be described by nine features. Only four, not five, distance features are needed to provide four shift-invariant features, the distance of each 
of the four other scatterers from the first scatterer. These four distance features are used because, in general, the first scatterer is the most stable scattering center in the radar return. This stability is derived from the absence of intermediate and late-time returns consisting of multi-bounce and shadowed scatterers which are more aspect dependent and unpredictable. Notice, however, that with these four values, a linear combination of two of them can derive the distance between any two scatterers. Due to the simplicity of this linear combination, all distances between scatterers are readily accessed by a neural net if any should prove useful for class discrimination.

Table 2. DSC Model Estimation Results and Corresponding Features Extracted

\begin{tabular}{c|c|c|c|}
$\alpha_{0}$ & \multicolumn{2}{c}{$\begin{array}{c}\text { geometry } \\
\text { feature }\end{array}$} & $\begin{array}{c}\text { distance } \\
\text { feature }\end{array}$ \\
\hline-0.5 & -0.1308 & -0.5 & - \\
\hline-1 & -0.0460 & -1 & 0.0848 \\
\hline-1 & -0.0012 & -1 & 0.1296 \\
\hline 0.5 & 0.0447 & 0.5 & 0.1755 \\
\hline-0.5 & 0.1050 & -0.5 & 0.2358 \\
\hline
\end{tabular}

It is obvious that a model order of five will not capture all of the scattering centers in as complex an object as an aircraft, where dozens of localized scatterers of various geometries could certainly be predicted and perhaps modeled. However, if the largest five scatterers estimated do not completely model aircraft, they may still sufficiently represent the object class for this data set. This principle is very important in object recognition. Simply stated, the criteria is not that scattering is precisely modeled, but rather, that the features are

1. reasonably stable across all samples of one class and

2. discriminatory between samples of different classes.

To demonstrate the viability of feature extraction based on the DSC model's range and type parameters, a typical object classification experiment was performed. The experimental data contained 1204 direct range measurements of four model aircraft of similar size and shape at 0 degrees elevation and from 0 to 30 degrees azimuth. After implementing DSC model feature extraction on this data, a fully-connected, two-layer neural net obtained over $98 \%$ classification accuracy. Furthermore, DSC model feature extraction gave an approximate $85 \%$ reduction in the number of features required to characterize objects compared to the numerous range bin magnitudes used in template matching approaches. As expected, this feature efficiency greatly reduces computer memory and processing time over the template-based approach. In addition, template formation usually ignores the phase information contained in the phase history of the radar measurement. Conversely, DSC modeling takes full advantage of the phase information. 


\section{ACKNOWLEDGMENTS}

We thank Dr. Lee Potter and Mike Gerry of The Ohio State University for sharing both algorithms and discourse regarding their radar scaltering center estimation research.

\section{REFERENCES}

[1] H.-J. Li and S.-H. Yang, "Using range profiles as vectors to identify aerospace objects," IEEE Trans. Antennas Propagaf.,vol. 41, pp. 261-268, Mar. 1993.

[2] P. B. Silverstien, O. S. Sands and F. D. Garber, "Radar target classification and interpretation by means of structural descriptions of backscatter signals," IEEE AES Systems Magazine, pp. 3-7, May 1991.

[3] M. P. Hurst and R. Mittra, "Scattering center analysis via Prony's method," IEEE Trans. Antennas Propagat., vol. 35, pp. 986-988, Aug. 1987.

[4] R. Carriére and R. L. Moses, "High resolution radar target modeling using a modified Prony estimator" IEEE Trans. Antennas Propagat., vol. 40, pp. 13-18, Jan. 1993.

[5] J. J. Sacchini, W. M. Steedly and R. L. Moses, "Two-dimensional Prony modeling and parameter estimation," IEEE Trans. Signal Processing, vol. 41, pp. 3127-3137, Nov. 1993.

[6] Y. Hua and T. K. Sakar, "Matrix Pencil method for estimating parameters

of exponentially damped/undamped sinusoids in noise," IEEE Trans. Acoustic, Speech, Signal Process., vol. 38, pp. 814-824, May 1990.

[7] J. B. Keller, "Geometric theory of diffraction," J. Opt. Soc. Amer., pp. 116-130, Jan. 1962.

[8] R. G. Kouyoumiian and P. H. Pathak, "A uniform geometrical theory of diffraction for an edge in a perfectly conducting surface," Proceedings of the IEEE, vol. 62, PP. 1448-1461, Nov. 1974.

[9] A. Moghaddar, Y. Ogawa, and E. K. Walton, "Estimating the time-delay and frequency decay parameter of scattering components using a modified MUSIC algorithm," IEEE Trans. Antennas Propagat., vol. 42, pp. 1412-1418, Oct. 1994.

[10] L. C. Potter, D.-M. Chiang, R. Carriére, and M. J. Gerry, "A GTD-based parametric model for radar scattering," IEEE Trans. Antennas Propagat., vol. 43, pp. 1058-1067, Oct. 1995.

[11] D.-M. Chiang, "Parametric signal processing techniques for model mismatch and mixed parameter estimation," Ph.D. dissertation, The Ohio State Univ., Columbus, 1996.

[12] R. Prony, "Essai experimental et analytique sur les lois de la dilatabilite de fluides elastiques et sur celles de la force expansion de la vapeur de l'alkool, a differents temperatures, " $J$. L'Ecole Polytech, vol. 1, pp. 24-76, 1795.

[13] D. R. Wehner, High Resolution Radar. Boston: Artech House Inc., 1987.

[14] E. F. Knott, ]. F. Shaeffer, and M. T. Tuley, Radar Cross Section. Boston: Artech House Inc., 1993.

[15] W. G. Carrara, R. S. Goodman, and R. M. Majewski, Spotlight Synthutic Aperture Radar. Boston: Artech House Inc., 1995.

[16] C. W. Therrien, Decision Estimation and Classification. New York: Wiltey, 1989. 